Bull. Fac. Sei, , Ibaraki Univ., Math., No. 4, 1972

\title{
Notes on Duality Theorems
}

\author{
Ryûki MATSUDA*
}

$\S 0$.

In $\S 1$, we improve [2]. In $\S 2$, we give a counter example for the Tannaka duality of topological groups. Results of these sections are after Roeder [3]. In $\S 3$, we supplement [1]. The author thanks Mr. Hirabuki for his encouragement.

$\S 1$.

1. The case [2], 3. If only $G_{2}$ satisfies the Tannaka duality, $\theta$ is a homeomorphism. Conversely, if $\theta$ is a homeomorphism for $G_{1}=Z$ (the rational integers), $G_{2}$ satisfies the Tannaka duality.

2. The case [2], 5. If only $G_{2}$ satisfies the generalized Tannaka duality, $F$ and $\Psi_{0}$ are canonically homeomorphic. Conversely, if $F$ and $\Psi_{0}$ are canonically homeomorphic for $G_{1}=Z, G_{2}$ satisfies the generalized Tannaka duality.

3. The case [2], 7. If only $\mathrm{g}_{2}$ satisfies the Tannaka duality, $\theta: F$ $\rightarrow \Psi$ is a bijection. Conversely, if $\theta$ is a bijection for $g_{1}=K, g_{2}$ satisfies the Tannaka duality.

The same holds for the generalized Tannaka duality.

4. The case [2], 9. If only $G_{2}$ satisfies the Tannaka duality, $\theta$ : $F \rightarrow \Psi$ is bijective.

$\theta$ is injective for any $G_{1}$, if and only if $G_{2}$ is linear.

The same holds for the generalized Tannaka duality.

\section{\&2.}

1. Let $G$ be a topological group. For any positive integer $n$, we define ${ }^{n} G$ the intersection of kernels of all continuous n-dimensional complex representations of $G$. Let $V$ be a neighbourhood of 1 in $G$.

Received August 15, 1971.

*Department of Mathematics, Faculty of Science, Ibaraki University, Mito, Ibaraki, Japan. 
If $G$ satisfies the generalized Tannaka duality [4], then we have ${ }^{n} \mathrm{G} \subset \mathrm{V}$ for all sufficiently large $\mathrm{n}$.

2. We see, using the fact of 1 , that the example by Roeder ([3], 4 ) is a counter example for the generalized Tannaka duality (accordingly for the Tannaka duality [5]).

\section{$\S 3$.}

It is proved in [1] that the universal domain of characteristic 2 does not satisfy the Tannaka duality as Lie algebra. Let $p$ be any positive prime number. We will prove moreover that any field $K$ of characteristic $p$ does not satisfy the Tannaka duality nor the generalized Tannaka duality.

Proof. Let $A \in \mathrm{gl}(n, K)$. We get a representation $\rho_{\Delta}$ of the Lie algebra $K$ by setting $\rho_{A}(x)=x A$ for $x \in K$. All representations of $K$ are of this form. We denote $K^{\times}$the set of representations of $K$. We get a special representation $\zeta$ of $K^{\times}$by setting $\zeta\left(\rho_{A}\right)=A^{p}$ for $\rho_{A} \in K^{\times}$. Now assume that the canonical map $\Phi: K \rightarrow K_{0}^{\times \times}$to the set of special representations of $K^{\times}$is bijective. We have $\Phi(x)=\zeta$ for some $x \in K$. Setting

$$
M=\left[\begin{array}{ll}
1 & 1 \\
0 & 1
\end{array}\right]
$$

we get the contradiction:

$$
\left[\begin{array}{ll}
x & x \\
0 & x
\end{array}\right]=x M=\rho_{M}(x)=\Phi(x)\left(\rho_{M}\right)=\zeta\left(\rho_{M}\right)=M^{p}=\left[\begin{array}{ll}
1 & 0 \\
0 & 1
\end{array}\right] .
$$

\section{References}

[1] R. Matsuda, A counter example for the duality of Lie algebras, Bull. Fac. Sci., Ibaraki Univ., Math., 2-2(1970), 1-3.

[2] R. Matsuda and K. Sato, A topic on duality theorems, Bull. Fac. Sci., Ibaraki Univ., Math., 2-1(1969), 15-18.

[3] D. Roeder, A characterization of unitary duality, Trans. Amer. Math. Soc., 148 (1970), 129-135.

[4] K. Sugita and M. Sugiura, On a certain type of duality theorem, Sci. Papers of the College of Gen. Edu., Univ. of Tokyo, 18(1968), 115-124.

[5] M. Sugiura, Some Remarks on duality theorems of Lie groups, Proc. Japan Acad., 43(1967), 927-931. 